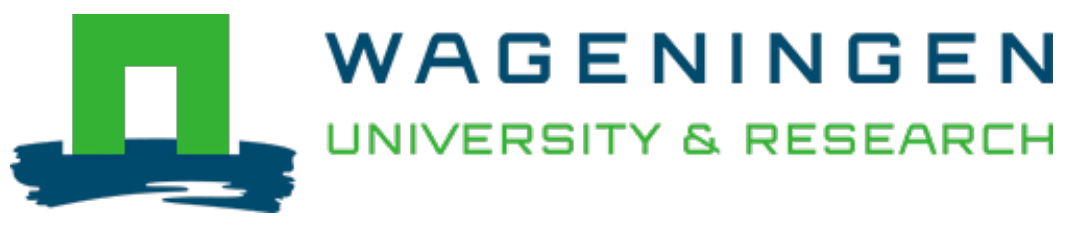

\title{
Systems and Precision Medicine in Necrotizing Soft Tissue Infections
}

Necrotizing Soft Tissue Infections

Martins dos Santos, Vitor A.P.; Hardt, Christopher; Skrede, Steinar; Saccenti, Edoardo

https://doi.org/10.1007/978-3-030-57616-5 12

This article is made publicly available in the institutional repository of Wageningen University and Research, under the terms of article $25 \mathrm{fa}$ of the Dutch Copyright Act, also known as the Amendment Taverne. This has been done with explicit consent by the author.

Article $25 \mathrm{fa}$ states that the author of a short scientific work funded either wholly or partially by Dutch public funds is entitled to make that work publicly available for no consideration following a reasonable period of time after the work was first published, provided that clear reference is made to the source of the first publication of the work.

This publication is distributed under The Association of Universities in the Netherlands (VSNU) 'Article 25fa implementation' project. In this project research outputs of researchers employed by Dutch Universities that comply with the legal requirements of Article $25 \mathrm{fa}$ of the Dutch Copyright Act are distributed online and free of cost or other barriers in institutional repositories. Research outputs are distributed six months after their first online publication in the original published version and with proper attribution to the source of the original publication.

You are permitted to download and use the publication for personal purposes. All rights remain with the author(s) and / or copyright owner(s) of this work. Any use of the publication or parts of it other than authorised under article $25 \mathrm{fa}$ of the Dutch Copyright act is prohibited. Wageningen University \& Research and the author(s) of this publication shall not be held responsible or liable for any damages resulting from your (re)use of this publication.

For questions regarding the public availability of this article please contact openscience.library@wur.nl 


\title{
Systems and Precision Medicine in Necrotizing Soft Tissue Infections
}

\author{
Vitor A. P. Martins dos Santos, Christopher Hardt, \\ Steinar Skrede, and Edoardo Saccenti
}

\section{Contents}

12.1 Introduction: The Case for a Systems and Personalized Approach

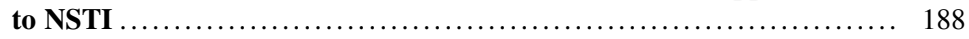

12.2 State of the Art: Systems, Precision and Personalized Medicine in NSTI 189

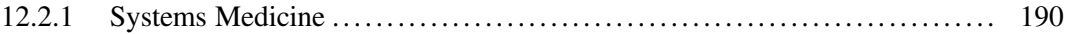

12.2.2 Challenges in Systems Medicine and How to Tackle Them .............. 190

12.2.3 Precision and Personalized Medicine ............................ 192

12.3 Big Data, Machine Learning and Deep Learning in Systems Medicine 193

12.3.1 Big Data Definitions and Characteristics ......................... 193

12.3.2 AI in Systems Medicine .................................... 194

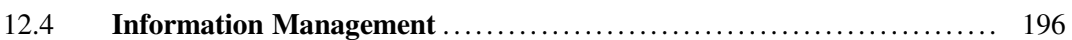

12.4.1 Personalized and Precision Medicine ............................... 196

12.4.2 The Case of NSTI ................................................ 197

12.4.3 Integrating Heterogeneous Data with FAIR Principles .................. 197

12.4.4 Laying the Basis for Computer-Assisted Decision Support .............. 198

V. A. P. Martins dos Santos $(\bowtie)$

Laboratory of Systems and Synthetic Biology,

Wageningen University and Research, Wageningen, The Netherlands

Lifeglimmer GmbH, Berlin, Germany

e-mail: vitor.martinsdossantos@wur.nl

C. Hardt

Lifeglimmer GmbH, Berlin, Germany

S. Skrede

Department of Clinical Science, University of Bergen,

Bergen, Norway

Department of Medicine, Haukeland University Hospital, Bergen, Norway

E. Saccenti

Laboratory of Systems and Synthetic Biology,

Wageningen University and Research, Wageningen, The

Netherlands 
12.5 Clinical Decision Support Systems for Soft Tissue Infections ............199

12.5.1 The Need to Enhance Medical Decisions ...................................... 199

12.5.2 What Are CDSS What Is Their Use ...................................

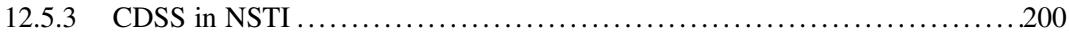

12.5.4 Current and Future Developments in Relation to Dedicated CDSS for NSTI . 201

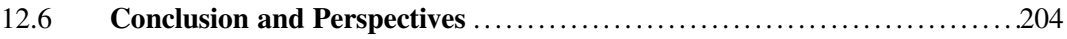

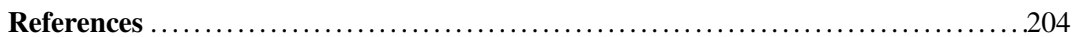

\section{Abstract}

Necrotizing soft tissue infections (NSTI) are multifactorial and characterized by dysfunctional, time dependent, highly varying hyperto hypo-inflammatory host responses contributing to disease severity. Furthermore, host-pathogen interactions are diverse and difficult to identify and characterize, due to the many different disease endotypes. There is a need for both refined bedside diagnostics as well as novel targeted treatment options to improve outcome in NSTI. In order to achieve clinically relevant results and to guide preclinical and clinical research the vast amount of fragmented clinical and experimental datasets, which often include omics data at different levels (transcriptomics, proteomics, metabolomics, etc.), need to be organized, harmonized, integrated, and analyzed taking into account the Big Data nature of these datasets. In this chapter, we address these matters from a systems perspective and yet personalized approach. The chapter provides an overview on the increasingly more frequent use of Big Data and Artificial Intelligence (AI) to aggregate and generate knowledge from burgeoning clinical and biochemical information, addresses the challenges to manage this information, and summarizes current efforts to develop robust computer-aided clinical decision support systems so to tackle the serious challenges in NSTI diagnosis, stratification, and optimized tailored therapy.

\section{Keywords}

Artificial intelligence $\cdot$ Big data $\cdot$ Clinical decision support systems · Deep learning
Information management · Personalized medicine $\cdot$ Semantic technologies

\section{Highlights}

- Systems, precision and personalized medicine approaches are methods to facilitate and improve contemporary diagnosis, accurate stratification, and optimized tailored therapy in NSTI.

- Big Data analytics and artificial intelligence are increasingly allowing to uncover disease mechanisms, giving a basis for patient stratification.

- We have established a prototype of an advanced platform for personalized medicine in NSTI according to FAIR principles.

- We have developed the basis for a clinical decision support system in NSTI.

\subsection{Introduction: The Case for a Systems and Personalized Approach to NSTI}

NSTI are complex multifactorial diseases that can be caused by a variety of microbes. They are frequently complicated by septic shock and multi-organ failure. Despite modern medicine, the mortality is high, often exceeding $25 \%$, and amputation is required in up to $15 \%$ of the cases (Peetermans et al. 2020). Most patients affected are individuals with co-morbidities, e.g. cardiovascular diseases and diabetes mellitus, but some patients are young immunocompetent individuals. The fulminant, often rapid course of these invasive infections (Kittang et al. 2010), demands early diagnosis and immediate intervention. However, misdiagnosis and subsequent doctor's delay is frequent (Goh et al. 2014), due 
to discrete and unspecific initial symptoms, scarce clinical findings and lack of biomarkers in early stages of many NSTI.

The cornerstones in the treatment of NSTI are early aggressive surgical debridement and appropriate antimicrobial therapy. Frequently there is a need for advanced supportive measures. There is clearly an urgent need for refined bedside diagnostics and novel, targeted treatment options to improve outcome in these patients. Because of the multifactorial nature of NSTI and sepsis, it is becoming increasingly clear that individualized approaches are required to improve outcome of these patients. These infections are characterized by dysfunctional, highly varying hyper- to hypo-inflammatory, host responses contributing to disease severity (Goldstein et al. 2007; Sarani et al. 2009; Peetermans et al. 2020). Thus, individualized therapeutic strategies targeting both pathogen as well as the host response have great potential, but require patient stratification based on disease signatures.

Data are becoming available showing the power of omics in identification of disease signatures (Davenport et al. 2016), as well as in providing the basis for patient stratification through large-scale analysis of clinical data (Hardt et al., in preparation), but fail to acknowledge the complexity of the host-pathogen interactions and the patient's individual responses to infection and its progression. In this chapter, we address these problems from a systems medicine perspective, provide an overview on the increasingly more frequent use of Big Data and Artificial Intelligence (AI) to aggregate, and generate knowledge from burgeoning clinical and biochemical information directing towards personalized approaches. Moreover, we address the challenges to manage this information and summarize current efforts to translate this information into actionable knowledge through the development of robust computer-aided clinical decision support systems (CDSS) to tackle the serious challenges in NSTI diagnosis, stratification, and optimized tailored therapy.

\subsection{State of the Art: Systems, Precision and Personalized Medicine in NSTI}

Current medical science is largely conducted under a reductionist paradigm, which involves the notion that complex phenomena like mechanisms underlying onset of infectious diseases, interactions between host and pathogens and disease progression may be better understood by breaking them down into smaller, simpler components (Ahn et al. 2006).

However, given the complexity of the conditions under study and the myriad of underlying factors, this often leads to biased focus and oversimplification (i.e. by focusing only on a handful of major factors with the biggest effect, while the sum of minor factors may be considerable) and generalization (i.e. assuming that a common causeeffect relationship applies equally in all cases).

Such simplifications and generalizations often limit our ability to understand how multiple variables interact with one another to create emergent effects and hamper not only our understanding of the disease, but more importantly, our capability of delivering better treatments. There is clearly a need to address health and disease from a systems perspective, that is, one that accounts for all factors and interactions. For instance, Chap. 12 illustrated the application of such a systems (biology) approach for the identification of potential biomarker sets in NSTI. This entailed the uncovering of metabolite-metabolite association networks and analysis and deployment of machine learning methods (Afzal et al. 2020). These findings would not have been possible with a traditional, reductionist approach of trying to identify potential biomarkers by standard approaches that are based, e.g. on simple univariate analysis of datasets, as these fail to account for the interrelatedness existing among genes, proteins, and metabolites that behave in an orchestrated way for what concerns regulation, transcription, and translation (Rosato et al. 2018). 
Fig. 12.1 Schematic illustration of the core differences between reductionism and systems science, when analyzing the properties of a system (Tillmann et al. 2015). Licensed under Creative Commons

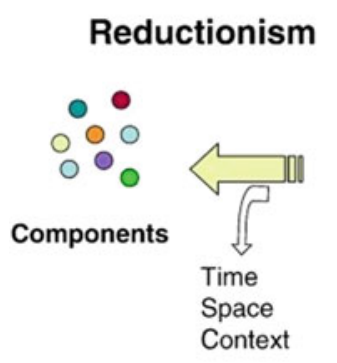

Medical Treatments

Disease-driven Aimed for normalcy (normal range) Additive

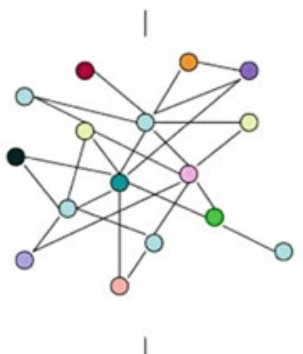

Systems Science

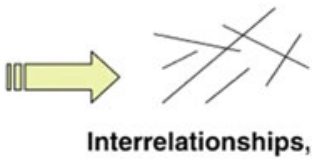

Dynamics

\section{Medical Treatments}

Individualized

Multidimensional use of drugs

Time-sensitive

Space-sensitive

Synergistic

\subsubsection{Systems Medicine}

Building upon the uncovering of biological mechanisms as above and going beyond that, "Systems medicine" applies systems biology approaches to medical research and medical practice. Its objective is to integrate a variety of biological/medical data at all relevant levels of cellular organization using the power of computational and mathematical modelling, to enable understanding of the pathophysiological mechanisms, prognosis, diagnosis, and treatment of disease (Auffray et al. 2010). Systems Medicine involves iterative and reciprocal feedback between the clinical practice and the research carried on with computational, statistical, and mathematical multiscale analysis and modelling at both the epidemiological and individual patient level (www.easym.eu).

This new paradigm of Systems Science and Medicine complements the traditional reductionist approach (as exemplified in Fig. 12.1) and, ideally, leads to the identification of mechanisms related to disease pathophysiology, selection of novel drug targets and biomarkers, patient stratification, risk assessment, and optimized therapy.

As described in Chap. 1, the systems medicine INFECT project (https://permedinfect.com/ projects/infect/) has generated comprehensive knowledge of diagnostic features, causative microbial agents, treatment strategies, and pathogenic mechanisms (host and bacterial disease traits and their underlying interaction networks) by using the largest thus far patient cohort in the world. Also, INFECT has proven the value of systems medicine approaches in acute infectious diseases to achieve improved diagnostics and therapeutics to improve patient disease outcome. In particular, the novel understanding of the disease mechanisms has resulted in changed clinical practice related to antibiotic usage as well as use of immunomodulatory treatments (Bergsten et al. 2020; Madsen et al. 2018) (see also Chaps. 7, 8, and 9).

However, the insights gained also underscore the need for patient stratification and implementation of tailored therapy. Despite considerable progress, there are substantial hurdles to be overcome related to the difficulties in accounting for patient's individual conditions, or intrinsic to Systems Medicine itself.

\subsubsection{Challenges in Systems Medicine and How to Tackle Them}

The recent transition to data-rich (i.e. molecular characterization) applications, which followed the omics revolution with the advent of high throughput genomics, transcriptomics, metabolomics, and all other omics disciplines, 
have enabled Systems Medicine approaches (Noble 2008). However, this data deluge is at the same time an opportunity and a challenge. An opportunity because it allows patient phenotyping at different levels in an unprecedented manner, and a challenge because of the heterogeneity observed in both data and patients.

There is an inherent data heterogeneity which arises when different data types and different data resources are integrated. This poses challenges for both data handling and analysis which can be addressed by vertical integration, i.e. when different data informs on the same biological level, or by horizontal integration, i.e. when multiple datasets inform on the same biological level (Berthold et al. 2010). Both cases can be handled, using FAIR data approaches (Findable, Accessible, Interoperable, Reusable) and semantics technologies (Berthold et al. 2010) but, as a matter of fact, there are other inherent sources of variability, which poses even greater challenges to Systems medicine approaches.

Humans exhibit a great phenotypic diversity, which originates from the complex interplay of genetic, epigenetic, and environmental factors, that affects both disease manifestations and responses to therapy (Assfalg et al. 2008; Bernini et al. 2009). Nonetheless, intra-individual variability is less than half the inter-individual variability, making personalized medicine possible (Hughes et al. 2015; Gruden et al. 2012). Under this light, statistical and computational characterization of individual and inter-individual variability is pivotal to the deployment of systems and personalized medicine approaches, which will allow both higher sensitivity and specificity of personalized assays and substantial new insights into health and disease.

Characterization of phenotypic diversity in both health and disease and underlying biomolecular mechanisms should be carried out at different levels. Examples could be developing new statistical and computational methods, to exploit the wealth of information obtainable at all omics levels, or by developing and deploying multiscale approaches to model how processes occurring at widely different scales integrate, results in the phenotypic variability observed in humans.
Multiscale modelling is another way to approach the problem of phenotypic variability. This includes bridging molecular and physiological processes, even taking place at very different time and spatial scales (Meier-Schellersheim et al. 2009). This could facilitate a better understanding of the biology of health and disease, allowing us to tailor models to individual patients (e.g. genome-scale metabolic model constrained by patient-specific data).

A key challenge is to select measurements and data collected at the small scales and combine them into informative metrics to be transferred to a higher level. This is the realm of metamodelling, which is the statistical approximations or predictions of the relationships between the various model components. Meta-modelling has been proposed as an efficient solution to link models obtained at different scales, to link modelling results and measured data, and to identify the most important metrics determining system functionality at the various levels (Tøndel et al. 2012).

Among statistical approaches derived from multivariate analysis, multi-way analysis has been proposed to retain the block-wise structure of temporal data originating from nonlinear dynamic models used to describe the systems at different levels (Tøndel et al. 2012). However, other solutions can be hypothesized that involve other component methods: some examples are principal component regression (Jolliffe 1982) or partial least square regression (Wold and Eriksson 2001) that can be used to model the complex relationships between input parameters and model outputs of nonlinear dynamic models likely with embedded procedure for variable selection to reduce dimensionality and complexity of the parameter space (Tøndel et al. 2012). Furthermore, parameter characteristics, such as distributional properties, covariance, and correlation patterns, could be inferred by stochastics methods designed to deconvolute correlative or noisy patterns: since signals can be separated from background biological variability in datadriven inference framework, this could ultimately lead to a better definition of phenotype characteristics. 


\subsubsection{Precision and Personalized Medicine}

Personalized Medicine builds upon Systems Medicine and is an emerging data-driven health care approach that integrates phenotypic, genotypic, epigenetic, lifestyle, and environmental factors unique to an individual. The goal of personalized medicine is to facilitate diagnosis, predict effective therapy, and avoid adverse reactions specific for each patient (Nimmesgern et al. 2017; Union 2015).

Precision medicine is the concept of tailoring disease treatment and prevention to account for differences in genetic, environmental, or even lifestyle factors specific to groups of people (Bresnick 2018). Precision medicine takes genetic and biochemical information unique to a group of patients and uses that information to develop more specific and streamlined medications or treatments. The goal is to ensure that each medication or treatment is best suited to treat the individual, resulting in decreased side effects and increased effectiveness (Hulsen et al. 2019).

Although the terms precision medicine and personalized medicine are sometimes used interchangeably, generally speaking, "precision medicine" seeks to create treatments that are applicable to groups of individuals who meet certain characteristics, whereas "personalized medicine" implies individualized treatments available for every unique patient (Erikainen and Chan 2019).

Precision and personalized medicine are in their infancy in infectious diseases, in particular acute diseases such as sepsis and NSTI (Lazăr et al. 2019). As the patient populations in severe infections are highly heterogeneous due to hostmicrobe interactions governed by different host factors and pathogens driving unique pathogenic mechanisms, personalized and precision medicine approaches may prove crucial. It is recognized that a dysregulated host response to infection is directly linked to severity and outcome of severe infections, such as sepsis and NSTI. Furthermore, the response profiles/disease signatures can be highly variable, ranging from hyper- to hypo-responses with different mediators involved (Hotchkiss and Karl 2003; Anaya et al. 2005; Huang et al. 2011; Thänert et al. 2019).

The identification of disease signatures of value for patient classification studies in welldefined patient cohorts has been undertaken, exploring host responses, pathogen profiles, and their association with disease outcomes (Chella Krishnan et al. 2016; Thänert et al. 2019). However, as for all biomarkers (see Chap. 12), these findings must be validated by use of other patient cohorts, as stated in a landmark position paper by the European Society of Clinical Microbiology and Infectious Diseases (Rello et al. 2018). Accordingly, to advance on precision medicine of NSTI and sepsis, two current multinational projects have been designed and implemented (PerMIT and PerAID, www.permedinfect.com). These projects build upon the unique resources created in the INFECT project, including clinical expertise, patient registry, biobank, multi-omics data, identified candidate disease signatures, data stewardship resources, and basic science experimental model systems.

These resources are currently being used to test data-driven working hypotheses through advanced preclinical and clinical studies combined with Big Data integration and information technology solutions, to develop patient stratification schemes allowing for individualized therapy in NSTI. The identified pathogenic mechanisms and biomarkers linked to particular NSTI disease signatures and clinical outcome are being further validated in sepsis patients, a condition that, just like NSTI, is defined by the host's response to an infection. A major strength of these on-going studies is that they resort on well-defined patient cohorts, allowing for robust conclusions linking host response signatures and pathogenic mechanisms to clinical outcomes encouraging translation of these findings into future patient handling measures.

Interestingly as well, these two synergistic projects envision that some stratification and clinical trial designs will be shared for NSTI and sepsis, whereas others will target more strictly defined patient subgroups. For example, sepsis patients with an immunosuppressive response 
profile might be targeted for an immune boosting therapy, whereas NSTI and sepsis patients with a hyperinflammatory profile will benefit from a suppressive agent.

Undoubtedly, such insights will allow for effective tailored treatments and development of new tools and concepts for future clinical trials. Crucially, however, for this potential to be fulfilled, some further challenges have to be tackled, in particular with regard to the handling of information and translation to actionable knowledge.

Indeed, a key challenge in the personalized/ precision medicine field is the vast amount of fragmented clinical and experimental datasets that need to be organized, harmonized, and integrated in order to achieve clinically relevant results and to guide preclinical and clinical research. This is also essential to enable fruitful Big Data analytics to yield meaningful insights, as well as for development of Clinical Decision Support Systems (CDSS) to assist effectively and efficiently in bedside decision.

\subsection{Big Data, Machine Learning and Deep Learning in Systems Medicine}

\subsubsection{Big Data Definitions and Characteristics}

Big Data and the development of techniques to handle it have the potential of enhancing our ability to probe and understand which parts of the biological machinery underlying the normal functioning of the organism that may be or become dysfunctional, given the pathophysiology of a condition (Hulsen et al. 2019).

Big Data are characterized by the so-called four V's (Schroeck et al. 2012), which stands for volume, variety, velocity, and variability.

1. Volume refers to the size of data, where size indicates the physical occupancy of data files. In Big Data applications exabytes, zettabytes, and even higher amounts of data need to be handled simultaneously. For what concerns systems medicine applications, the volume of the data is likely to be much smaller.

2. Variety refers to the heterogenous nature and sources of data. In the biomedical field the different types of data that can be collected, mined, and analyzed are virtually endless. Data comes not only in form of health records, clinical data, to which data from omics measurements can be added but also as medical imaging, including X-rays, CT, or MRI, or images recorded on tissue samples, e.g. tissue biopsies. These different data sources need to be handled and integrated to be properly analyzed, taking into account there are structured data (e.g. data stored in excel format) and unstructured data (like doctor's notes). This is usually one of the most challenging tasks.

3. Velocity refers to rate of data sampling and acquisition. Standard clinical measurements and omics data are usually static or acquired at a (very) low sampling rates. Differently, health monitoring systems, including, but not limited to, smartphones, smart watches, smart bracelets/wristbands, connected sensors, and wearable devices, enable continuous monitoring of patient data by sensing and transmitting measurements such as heart rate, blood pressure, body temperature, respiratory rate, chest sounds, and electrocardiogram (Vitabile et al. 2019) at high frequency, creating a flow of data that often needs to be processed "on-thefly."

4. Variability concerns the quality of the data acquired and their inconsistency. For biomedical and healthcare applications, data quality is a very critical aspect, because erroneous information can lead to erroneous diagnosis and treatment. The problem of obtaining quality data is complex and cross-disciplinary. Over the years, several organizations have contributed to defining the quality of various products and services and identifying ways of measuring such quality (Brighi 2018). Handling and management of data inconsistency, such those introduced by missing data, 
is of paramount importance for the exploitation of Big Data.

Accordingly, Big Data are high-volume, highvelocity and/or high-variety information assets that demand cost-effective, innovative forms of information processing that enable enhanced insight, decision making, and process automation. This definition (www.gartner.com/it-glos sary/big-data/), albeit stated in the context of informatics, summarizes the challenges and the gain that Big Data present and can offer to Systems Medicine.

The ultimate goal of the collection and use of Big Data in Systems Medicine is the possibility of obtaining better description of both health and disease profiles and to use them to build integrative models that can be used to predict disease onset and progression and to tailor better treatment for each patient. Under this light, Big Data approaches are fundamental for the development of Precision medicine, which aim to integrate phenotypic, genomic, epigenetic, and environmental factors unique to an individual to facilitate diagnosis, predict effective therapy, and avoid adverse reactions specific for each patient. Thus, precision medicine needs to operate on different scales to gain other insights into health and disease, utilizing and integrating data from cells, tissues, organs, and ecosystems, e.g. those constituted by microbial communities (Stacy et al. 2016).

\subsubsection{Al in Systems Medicine}

Artificial intelligence encompasses the use of software and algorithms to emulate the human cognition in the analysis of complex medical data.

AI is being successfully employed in several medical fields. Models have been constructed that enable to distinguish high-risk breast lesions (HRL) diagnosed with image-guided needle biopsy that require surgical excision from HRLs that are at low risk for upgrade to cancer at surgery (Bahl et al. 2018) or able to detect pneumonia using chest X-rays with an accuracy level exceeding practicing radiologists (Rajpurkar et al. 2017). Using images, artificial intelligence approaches have been used to describe the impact of orthognathic treatment on facial attractiveness and age appearance (Patcas et al. 2019). A seminal study (Esteva et al. 2017) tested the performance of AI to distinguish keratinocyte carcinomas versus benign seborrheic keratoses and malignant melanomas versus benign nevi, training it on $>15,000$ biopsy-proven diagnostic images, against 21 board-certified dermatologists, and found AI to perform on par with all tested experts across both comparisons, and other studies confirmed AI ability in identifying melanoma from dermoscopic images with accuracy similar to that of specialists (Phillips et al. 2019, 2020).

There are few applications of Big Data and AI to systems medicine specific to NSTI. The management of NSTI is complex given that clinical presentation is highly variable and range from early sepsis with obvious skin involvement to minimal cutaneous manifestations with a disproportionate systemic response (Bosshardt et al. 1996). Classic signs like fever, diffuse crepitus, and shock are late signs: once large blisters and gangrene develop, the infectious process is already at an advanced stage (Bosshardt et al. 1996). NSTI treatment must be aggressive and rapid and essential elements of the treatment are resuscitation, antimicrobial therapy, surgical debridement, and supportive care (Anaya et al. 2005; Morgan 2010; Hakkarainen et al. 2014; Stevens and Bryant 2017), and constant monitoring is required to achieve fluid, electrolyte, and hemodynamic stability (Bosshardt et al. 1996). Hyperbaric oxygen treatment can also be used as adjunctive therapy when infections involve anaerobic bacteria, specifically the clostridial species (Bakker 2012).

For instance, AI could be applied to the analysis of microbiological findings. Identification of the etiological agents can assist infection control measures and antimicrobial therapy decision making, and may offer prognostic information (Anaya et al. 2005; Huang et al. 2011; Madsen et al. 2019). The practical applicability of AI methods for the analysis of microbiology finding to aid diagnostic testing has been postulated and discussed for the image analysis including Gram 


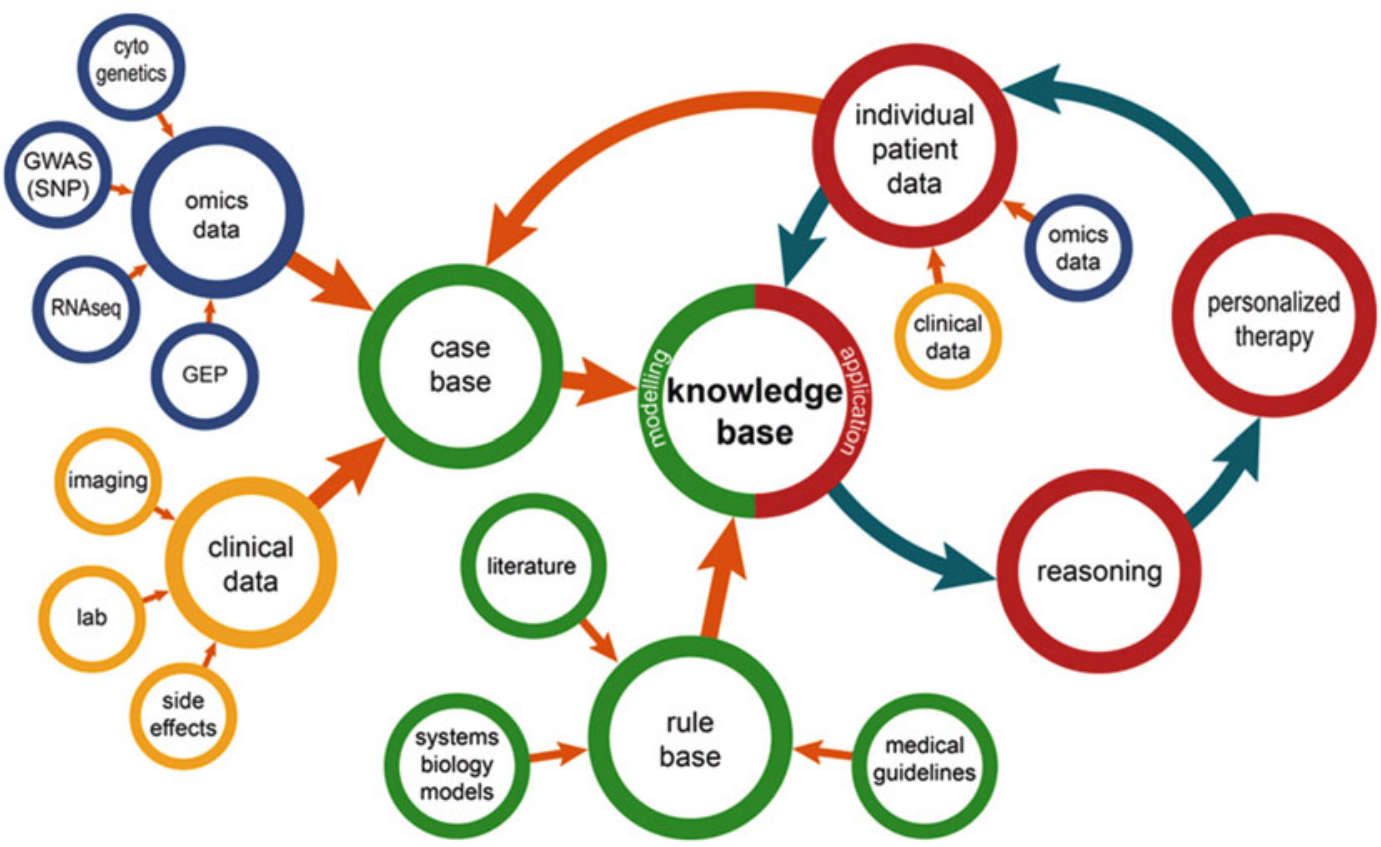

Fig. 12.2 Information management model for systems and personalized medicine. Figure from (Ganzinger and Knaup 2017), Licensed under Creative Commons

stains (Smith et al. 2020) which could be applied also to NSTI microbiology (see also Fig. 12.2). The role of radiologic imaging in diagnosis of NSTI is debated (Leichtle et al. 2016; Fernando et al. 2019) and several imaging options are available, such as plain radiographs, ultrasonography, computerized tomography (CT), and magnetic resonance imaging (MRI).

Although MRI images were previously not used in Big Data context or analyzed with AI methods, those could be easily applied to support and aid clinical decision. For instance, supervised algorithms could be trained on large datasets containing MRI images of patients with NSTI and related conditions, and these algorithms could be applied instantly, contextually with image acquisition and reconstruction, to provide guided diagnosis that could be integrated with other clinical information.

Kim et al. (2011) suggested that magnetic resonance imaging could be used to differentiate between necrotizing and non-necrotizing fasciitis. They compared MRI findings between the two groups and found that patients with necrotizing fasciitis had a significantly greater frequency of, among others, thick ( $\geq 3 \mathrm{~mm}$ ) abnormal fascial signal intensity on fat-suppressed T2-weighted images, low signal intensity in the deep fascia on fat-suppressed T2-weighted images. CT scanning has also been proposed (Hietbrink et al. 2016) but MRI scanning proves to have the highest sensitivity and specificity (Hietbrink et al. 2016).

Rakus-Andersson and Frey (Rakus-Andersson and Frey 2016) trained a modified neural network, to identify groups of NSTI patient with good prognosis of recovering without $\mathrm{HBO}$ compared to patients for which HBO could be beneficial, in such a way as to support clinical decision making. They used data from 13 patients admitted to the Blekinge County City Hospital in Karlskrona between 2006 and 2010. The input data consisted of clinical data (non-disclosed) and the results were satisfactory, with an accuracy of $92 \%$; however, these results were obtained on a very limited sample size and have not been crossvalidated.

The use of Big omics data in the clinical setting of NSTI to support diagnosis at the bedside is less immediate since these measurement 
platforms are usually not available in hospital settings: sample work-up, preparation, acquisition, data cleaning, and processing may require several days, which is a major hindrance, given the severity of NSTI in critically ill patients (Peetermans et al. 2020). A notable exception is the use of nuclear magnetic resonance, which requires minimal sample preparation to measure metabolomics profiles of blood and tissues samples collected in the operating theater and that can provide almost real-time information to the surgeons and to clinicians. Such a setting has been implemented at St. Mary Hospital in London (Nicholson et al. 2012).

The advent of new technologies is enabling real-time sequencing of large genomes and it is now possible to perform without delay sequencing and analysis of patient genetic information. Implementations of automated phenotyping and interpretation of genome sequencing by beadbased genome library preparation directly from blood samples and sequencing of paired 100-nt reads obtained $15.5 \mathrm{~h}$ and used for fast population-scale, provisional diagnosis of genetic diseases of infants in neonatal and pediatric intensive care units, have been reported (Clark et al. 2019). They reported a prospective $100 \%$ precision and a mean time saving of $22 \mathrm{~h}$ on diagnosis which subsequently affected treatment.

If Big omics data are less applicable for bedside decision support due to technical limitations, they are essential when defining strategies for patient stratifications and individualized therapy in NSTI. Indeed, the lack of stratification strategies (one of the cornerstones of precision medicine) is one of the biggest bottlenecks in NSTI management. Omics data from NSTI subjects (and possibly controls), such as transcriptomics, proteomics, lipidomics, and metabolomics profiles measured on blood and tissue biopsies, could be analyzed in relation to pathogens and clinical parameters using multivariate statistics, machine learning, and reverse engineering approaches to identify subgroups of patients demonstrating a survival benefit or favorable response to given treatments.

\subsection{Information Management}

\subsubsection{Personalized and Precision Medicine}

A key challenge in the fields of personalized and precision medicine is to organize, harmonize, and integrate the vast amount of fragmented clinical and experimental datasets, in order to achieve clinically relevant results and to guide preclinical and clinical research.

Typically, data of different sources such as electronic health record (EHR) systems, clinical research databases, or biomedical knowledge representations like (i.e. ontologies) have to be reviewed and prepared. Furthermore, an often overlooked weakness is the use of patient samples and/or omics data despite lack of linked clinical (meta)data, which greatly reduces the usefulness of the studies. Thus, information management is of paramount importance for systems and personalized medicine in research as well as clinical practice. To tackle these challenges, a variety of approaches have been suggested and implemented in different settings. One such model is a three-layer information technology architecture coupled to a cyclic data management approach, as proposed by Ganzinger and Knaup (2017). The generic high-level architecture of such a three-layer model entails:

\section{Data representation, \\ 2. Decision support, and \\ 3. User interface.}

As for data representation (layer 1), data and knowledge from different sources have to be prepared and made available for use in systems medicine. This includes data harmonization, transformation, and storage. In decision support (layer 2), the data and knowledge from layer 1 are processed by applying decision support approaches or systems biology models (see also Sect. 12.3 on Big Data). Systems medicine applications should be designed to assist and not replace human decisions. Consequently, the user interface for such an application (layer 3) must be 
carefully designed to support well-informed, reproducible, clinical decisions in an appropriate time frame. The core concept of this model is the knowledge base, which contains patient and disease-related data as well as formally represented knowledge including a variety of omics and biomedical data. The Decision Support is suggested as case-based and rule-based components (Ganzinger and Knaup 2017), see also Sect. 12.5 below pertaining Decision Support. This model is shown schematically in Fig. 12.2.

The complexity of the data management process depends on the level of heterogeneity prevalent in the data sources. To achieve sufficient case numbers it is often necessary to combine data on the same entity types from different sources. For example, in the multi-center approach hospitals decide to collaborate and share clinical data on a specific disease area to build a joint systems medicine application with a higher number of cases and therefore greater statistical power. In most cases, clinical documentation will not be based on identical specifications. Thus, in a harmonization step data definitions have to be evaluated for each attribute, both on a syntactic and semantic level.

\subsubsection{The Case of NSTI}

In the context of systems and personalized medicine in NSTI, the most comprehensive endeavor thus far is that being undertaken under the scope of a series of national and international research programs comprising teams from hospitals, medical research, academia, and industry (see Chap. 1 and www.permedinfect.com). These programs have built a platform for personalized medicine in acute infectious diseases with focus on NSTI and sepsis that form the basis for development of tools and concepts for refined diagnosis, patient stratification, and individualized treatment.

The various participating hospitals have made substantial standardization efforts, so that clinical partners provide patient cohorts, including both clinical registries and associated biobanks, which were used to populate a common information platform. This required previous ethical approvals and written informed consent from all patients [and to be updated and amended as need arises]. It required as well full compliance on Data \& Ethics governance, Good Clinical Practice guidelines, and European General Data Protection Regulations (GDPR) (Regulation 2016). The data platform developed includes systems to handle data in separated data domains and stores to protect data ownership and privacy requirements.

\subsubsection{Integrating Heterogeneous Data with FAIR Principles}

A key feature and goal of such multinational, multi-center projects like INFECT, PERAID, and PerMIT is to ensure that all data resources (both institutions' own and public) are properly integrated into a common framework. A distributed data lookup and retrieval service allow users to select relevant datasets for inclusion in analysis based on not only matching metadata but importantly clinical and biological relevance and this adds a vital level of quality control ensuring that only clinically relevant datasets are included. These encompass also demographics, clinical, and treatment aspects.

Strict clinical case definition criteria, as well as source of infection and severity scores such as simplified acute physiology score (SAPS) and sequential organ failure assessment (SOFA) are used for precise patient classifications, whereas microbiological results will be documented and allow for stratification according to etiology and virulence properties. A key component is the inclusion of heterogeneous omics and biochemical datasets, which are ultimately essential for establishing the mechanisms underlying clinical conditions. The carefully curated studies/datasets are integrated with a minimal data model for meta-data exposure using a Resource Description Framework (RDF) model (Lassila and Swick 1998), which is further empowered by using distributed search and indexing technologies such as Apache Lucene (Białecki et al. 2012). 
Data fusion and standardization is ensured throughout the use of specialized data fusion algorithms and well-functioning interactions among participating institutions.

The use of a RDF data model to manage data provenance and storage ensures that data comply with the FAIR guidelines for data management. Minimum information checklists are used to facilitate interpretation and reproducibility of results ensuring the inclusion of the relevant meta-data. All data types are (should be) to be represented in ontologies so that they can be integrated and remain interoperable as the types and size of data increase.

A systematic treatment of the data is ensured by the use of ontologies devoted to clinical and disease-related terms, such as Systematized Nomenclature of Medicine-Clinical Terms (SNOMED CT) (Cote 1986; Lussier et al. 1998). The structure of the underlying data resources is assessed using tools such as RDF2Graph (van Dam et al. 2015) to ensure that newly integrated datasets readily fulfill the quality standards and indicators.

Operation of the www.permedinfect.com platform enables both handling of heterogeneous data and the standardization of operating procedures, data, and models, as well as their storage and stewardship.

The structure and workflows comply with those required by the European Union on data management of research (European Commission 2016). The platform itself aligns with international data stewardship infrastructure such as the ESFRI ELIXIR (elixir-europe.org) or Nordic e-Infrastructure Collaboration (NeIC) program (https://neic.no/).

\subsubsection{Laying the Basis for Computer-Assisted Decision Support}

Any CDSS has to rely on a data platform (see, for instance, the HUNT platform, www.ntnu.edu/ hunt/data which inform the PerAID and PerMIT data projects), allowing different layers with different level of access and different levels of granularity at the data level.

At the first level, a close interaction between the clinicians and support personnel exists. The interaction on the electronic devices is optimized using the state-of-the-art technology to monitor and to optimize human-computer interfaces.

The second level prepares anonymized databases from level 1 using software that can process various types of data such as patient records, data collected in various forms, and biobank data to a format that can be included in the RDF databases through previous standardization. This software is to be used within the hospitals in a protected environment and only the anonymized data will be transferred to the second level. This data is then to be further digitalized and abstracted to the third level that will represent a statistical view on the original data.

The first and second level are unique for a particular clinical center, while data at the third level will be pooled across centers. The threelayered data structure helps to overcome the main limitations of medical support systems, which are usually not scalable and not interconnected. Data are translated to standard English vocabulary while keeping the original granularity of the data and third layer data will be shareable between different clinical sites.

This allows the CDSS to be scalable and used in other studied and countries by accounting for local language requirements (see Sect. 12.5). Then the third layer, containing highly processed data, can be made public and used to predict treatment outcomes and contributing to best practice management of the disease. The CDSS will therefore combine the information of all patients in the patient cohorts, thereby allowing for interoperability and scalability of the system. 


\subsection{Clinical Decision Support Systems for Soft Tissue Infections}

\subsubsection{The Need to Enhance Medical Decisions}

The quantity and quality of clinical data are expanding rapidly, including EHRs, disease registries, patient surveys, and information exchanges. Also, burgeoning amounts of data are becoming available for each patient, as is the increasing body of medical and basic sciences evidence. Hence, clinicians need tools to help them make rational decisions based on all these information (Wasylewicz and Scheepers-Hoeks 2019).

Big Data and digitalization, however, does not automatically mean better patient care. Several studies have shown that only implementing an EHR and computerized physician order entry (CPOE) has rapidly decreased the incidence of certain errors, introducing, however, many more (Magrabi et al. 2016). Therefore, high-quality clinical decision support is essential if healthcare organizations are to achieve the full benefits of EHR and CPOE.

In the current healthcare setting, healthcare providers often do not know that certain patient data are available in the EHR, do not always know how to access these data, do not have the time to search for them, or are not fully informed on the most current medical insights when facing a decision. It is said the healthcare providers often drown in the midst of plenty (Mamlin and Tierney 2016; Frost and Sullivan 2015; Bresnick 2016).

Moreover, decisions by healthcare professionals are often made in conjunction with/as part of direct patient contact, ward rounds, or multidisciplinary meetings. This means that many decisions are made in a matter of seconds or minutes. This way, their quality depends on the healthcare provider having all patient parameters and medical knowledge readily available at the time of the decision. Consequently, current decisions are still strongly confounded by experience and knowledge of the professional.
Computer technology and algorithms can assist by generating case-specific advice for clinical decision making. The systems used are usually referred to as CDSS, and are thus intended to improve health care delivery by enhancing medical decisions with targeted clinical knowledge, patient information, and other molecular or health information (Wasylewicz and Scheepers-Hoeks 2019).

\subsubsection{What Are CDSS What Is Their Use}

A traditional CDSS is comprised of software designed to be a direct aid to clinical decision making, in which the characteristics of an individual patient are matched to a computerized clinical knowledge base. Patient-specific assessments or recommendations are then presented to the clinician for a decision (Sutton et al. 2020). From a historical point of view, medication-related CDSS has been used for a long time and is still the most advanced (Garg et al. 2005). CDSSs today are primarily used at the point-of-care, for the clinician to combine their knowledge with information or suggestions provided by the CDSS. However, there are CDSS being developed with the capability to leverage data and observations otherwise unobtainable or uninterpretable by humans (Wasylewicz and Scheepers-Hoeks 2019).

Current CDSS often makes use of web-applications or integration with EHR and CPOE systems. They can be administered not only through desktop, tablet, smartphone but also through other devices such as biometric monitoring and wearable health technology. These devices may or may not produce outputs directly on the device or be linked into EHR databases (Dias and Paulo Silva Cunha 2018). The scope of functions provided by CDSS is vast, including diagnostics, alarm systems, disease management, prescription, drug control, and much more. CDSS ranges from personal digital assistant applications customized by a single clinician to multihospital mainframe-based surveillance systems meant to assure care for 


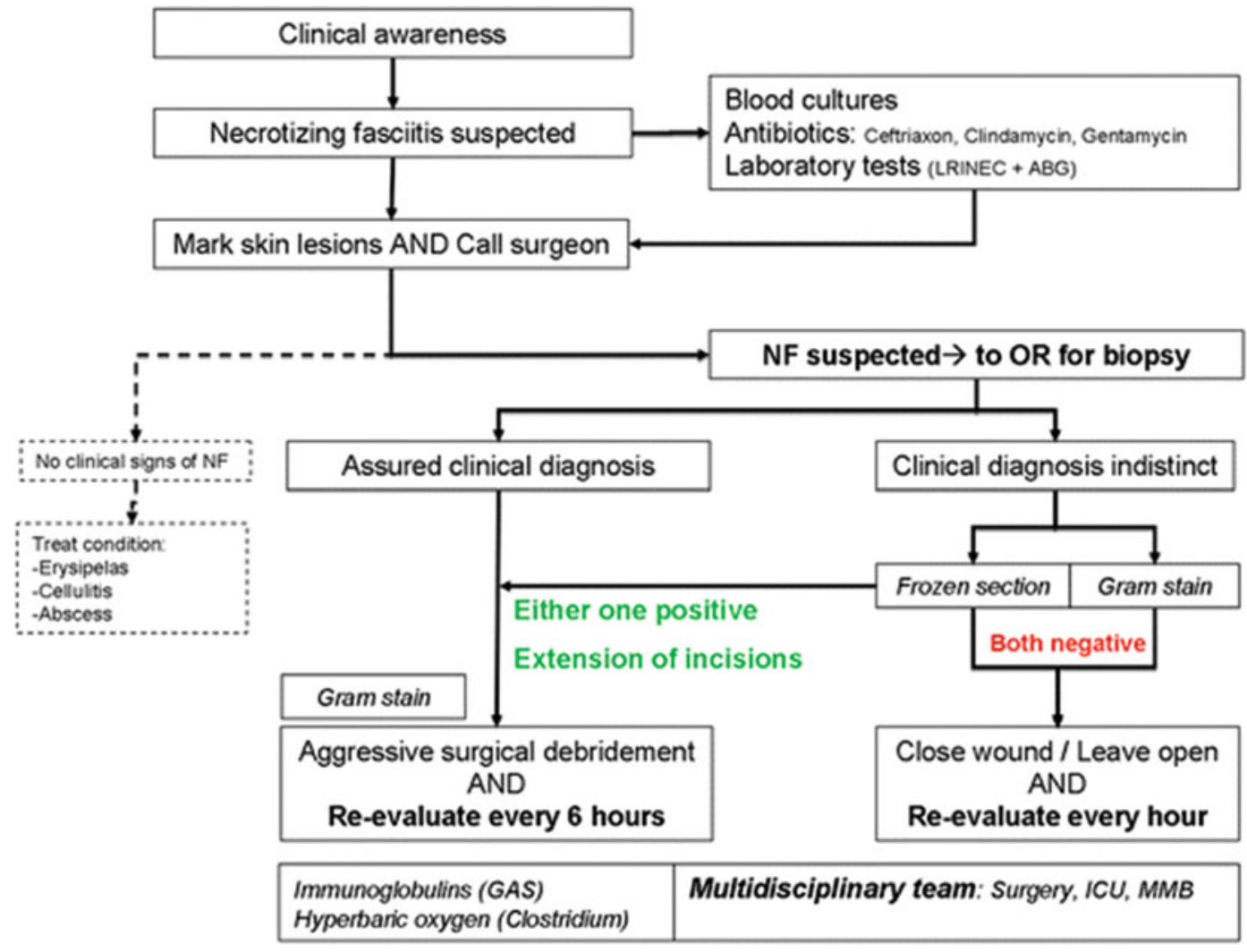

Fig. 12.3 Clinical algorithm for suspected fasciitis, as suggested by Hietbrink and co-workers (Hietbrink et al. 2016). The algorithm is used for gate specialties in patients with suspected necrotizing fasciitis. It consists of awareness, early surgical exploration, and early initiation of treatment. When incision biopsy is indicated, the patient

thousands of patients (Pusic and Ansermino 2004). They can manifest as computerized alerts and reminders, computerized guidelines, order sets, patient data reports, documentation templates, and clinical workflow tools.

Regarding the nature of the interaction with the clinician, CDSS may be categorized into those that entail solicited information (e.g. a clinician asking for specific advice for a given condition) or unsolicited information (deliver information or knowledge that beneficially can alter clinical decision making (Pusic and Ansermino 2004)). Such applications can be particular useful if CDSS builds upon robust and flexible data integration and includes a wide variety of data analytics. Such data analytics, in particular those based on AI, Machine Learning, and other is transported to the operation room for further treatment. Treatment and aftercare are multidisciplinary. Analysis of frozen section, microbiological findings, and biopsy could be supported by AI technologies. Figure and caption from Hietbrink et al. (2016), Licensed under Creative Commons

modelling tools, can be essential for rapid diagnosis, stratification, and assistance on decision regarding disease treatment and intervention strategies to be applied.

The benefits of CDSS, possible pitfalls, and evidence-based mitigation strategies to overcome have been published recently by Sutton et al. (2020) and discussed as well by Pusic and Ansermino (2004) and (Wasylewicz and Scheepers-Hoeks (2019).

\subsubsection{CDSS in NSTI}

Algorithmic procedures to handle NSTI patients have been defined (Hietbrink et al. 2016; Peetermans et al. 2020), see Fig. 12.3, but there 
are currently no CDSS or modules thereof dedicated to NSTI. Such modules or built-in tools would possibly be very useful for a range of uses, including those that could be included in CDSSs or applications routinely used by general practitioners, as these are often the first point of entry of potential patients, which are frequently misdiagnosed due to lack of familiarity with the disease (Goh et al. 2014).

Indeed, due to the fast progression of the disease, NSTI management requires fast decisions to determine the most appropriate course of action. Such decisions, or part of them, could be performed or supported by trained algorithms, e.g. aiding in the main diagnostic problem, that is to differentiate a lesion requiring surgery from a lesion for which conservative treatment will be sufficient. Furthermore, tasks like selection of antibiotic therapy and adjunctive therapy could be performed or optimized by dedicated algorithms, as suggested in the closely relating field of sepsis management (Komorowski 2020). Simple algorithmic procedures are already routinely applied in the management of NSTI. An example of this is the LRINEC (Laboratory Risk Indicator for Necrotizing Fasciitis) score which is generated from six routinely performed laboratory tests including the analyses of patients' C-reactive protein, white blood cell count, hemoglobin, sodium, creatinine, glucose. Wong et al. (2004) proposed a tool to distinguishing NSTI from the other severe soft tissue infections. However, recently its performance was evaluated in a prospective cohort study and data were displayed discouraging its use (Hsiao et al. 2020). An older study within the National Surgical Quality Improvement Program (NSQIP, USA) was used to determine data on the incidence, treatment, and outcomes of NSTIs (Mills et al. 2010). Partly on the basis thereof, a 30-day postoperative mortality risk calculator for patients with NSTI was developed and validated using a cohort of 1392 identified NSTI cases, of which $42 \%$ were female, median age was 55 years, and median body mass index was $32 \mathrm{~kg} / \mathrm{m}^{2}$ (Mills et al. 2010). Thirtyday mortality was $13 \%$. Seven independent variables were identified that correlated with mortality: age older than 60 years, functional status, requiring dialysis, American Society of
Anesthesiologists class 4 or higher, emergent surgery, septic shock, and low platelet count. The receiver operating characteristic area was 0.85 (95\% CI 0.82-0.87), reflecting a reasonably strong prediction. Using bootstrap validation, the optimism-corrected receiver operating characteristic area was 0.83 (95\% CI 0.81-0.86), which was used to develop an interactive risk calculator for future patients. Although not a CDSS, this correlation was nevertheless useful for stratification, according to the authors.

Another cohort study was reported (Hua et al. 2015) that included 109 patients with a confirmed diagnosis of NSTI, a median follow-up of 274 days (range 2-6135 days) and of which $31(28 \%)$ died. On multivariate analysis, independent risk factors of mortality were age older than 75 years, multifocal NSTI, severe peripheral vascular disease, hospital-acquired infection, severe sepsis, and septic shock on hospital admission. Although a retrospective cohort, which disallows a precise record of the delay between diagnosis and surgery, these analyses could help building information to develop a true CDSS and help clinicians stratify NSTI severity at clinical course onset.

A triple diagnostic procedure has been proposed to manage NSTI (Hietbrink et al. 2016) which combines the analysis of microscopic findings on tissue biopsies together with Gram staining to assess the presence, gram staining, characteristic arrangements, and morphology of microorganisms and analysis of fresh frozen sections to detect necrosis of the superficial fascia with fibrinous thrombi of arteries and veins located in the fascia. The algorithmic procedure is described in Fig. 12.3, in which many steps could be replaced or supported by AI- and Big Data-informed decision.

\subsubsection{Current and Future Developments in Relation to Dedicated CDSS for NSTI}

Specific efforts to develop and deploy innovative clinical support tools for patient stratification and bedside decisions suitable for the emergency and intensive care setting for NSTI are currently being 


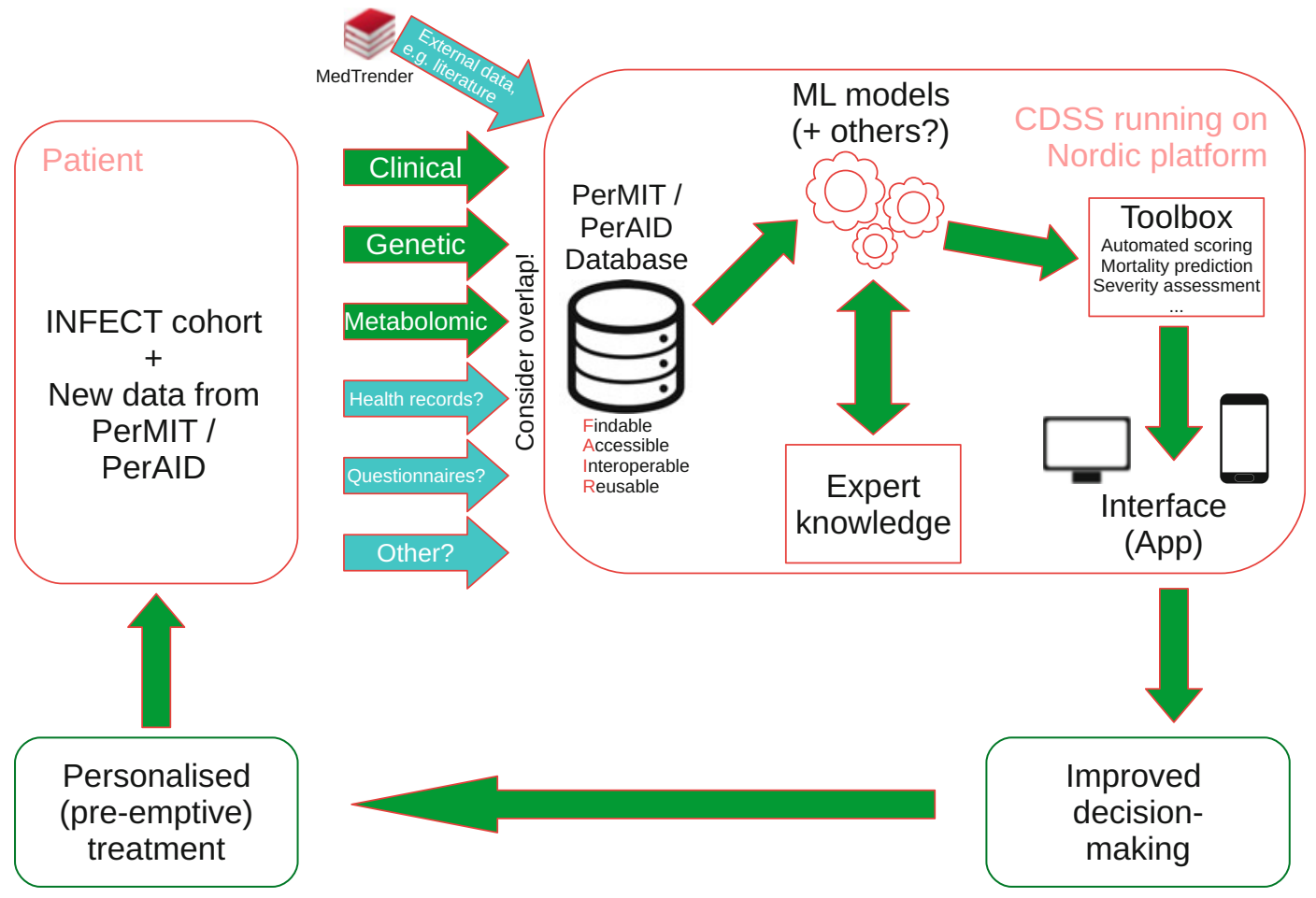

Fig. 12.4 Simplified representation of a CDSS for NSTI under development in the scope of the projects PerAID and PerMIT (Figure reproduced from permedinfect.com, author's copyright)

undertaken in a series of national and international research projects comprising teams from hospitals, medical research, academia, and industry (see permedinfect.com). One specific task is the development of Machine Learning and AI models for the prediction of different outcomes in NSTI, such as risk of sepsis, septic shock, or amputation, using a combination of clinical parameters including SAPS and SOFA severity scores and biomarkers that can be measured during the acute stages (e.g. in the emergency room), thereby enabling rapid bedside decisions.

The same data approach is being used to identify an optimized scoring of NSTI patients, to overcome some of sensitivity issues identified with the severity score LRINEC indicated above (Hansen et al. 2017).

Development of automatic calculations of such severity score is part of the basis for applications offering personalized decision support. Considering that different hospitals have their own sepsis alert system, such automatic calculations linked to the patients charts and sepsis alarm are considered as a means to achieve a more rapid and optimized identification of patients. This type of systems can help prioritizing severe cases and thereby reduce the clinical burden and efficient use of hospital beds.

An effective and efficient CDSS is of particular importance for the stratification of patients and personalized therapy in NSTI. Several dedicated modules are currently under development (www. permedinfect.com) for: (1) the integration of a variety of quantitative or qualitative models (i.e. statistical models, algorithms, etc.) to enable a CDSS to perform data analytics (see previous chapter and section on AI and Big Data above); (2) aggregation of reasoning processes from the domain and inference capabilities (e.g. rule-base and case-based systems) to handle the data/information (i.e. clinical and experimental parameters); and (3) user interfaces to match the need of practitioners in the clinic.

The software architecture model for the NSTIdedicated CDSS, the representation of interoperable clinical knowledge, and inference engine are 


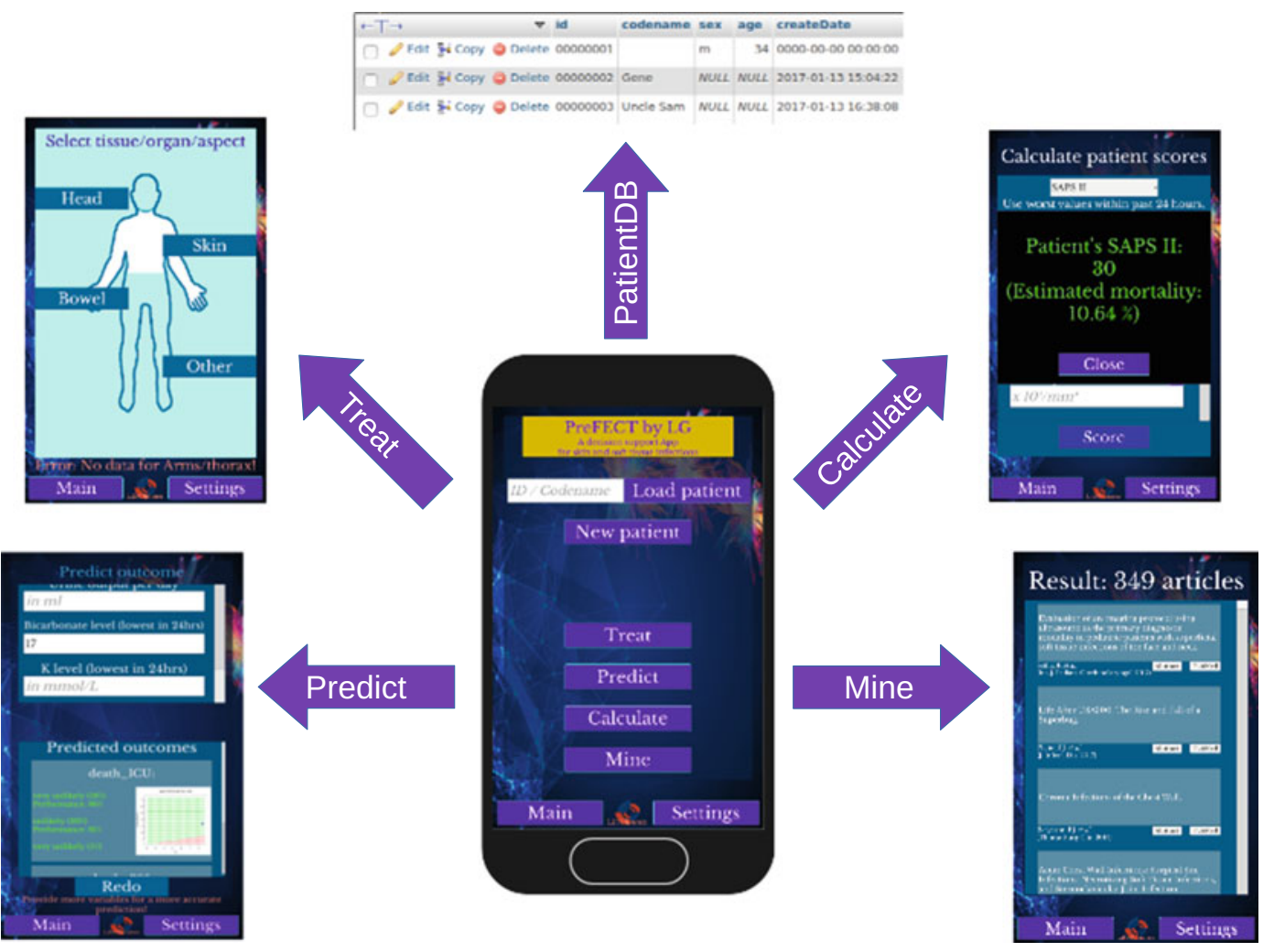

Fig. 12.5 Representation of a simple mobile app for use by clinicians as a front-end of a CDSS for NSTI (LifeGlimmer $\mathrm{GmbH}$ ). It enables data mining, querying databases, calculating specific scores, predicting outcomes and generally supports the clinician for decisions

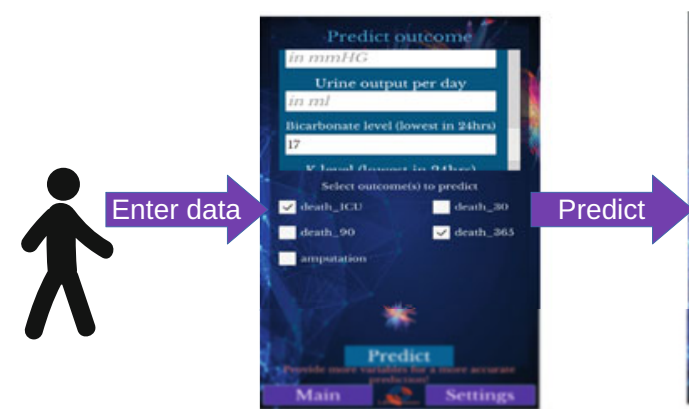

Fig. 12.6 Example of the use of the app as support to beside decision. Based on over 2000 current clinical parameters and by using Machine Learning algorithms (tested in the INFECT patient cohort), it predicts severity

being designed to form a base for a CDSS framework of wider applicability. The CDSS functionalities are being iteratively developed through requirement-adjustment-development-

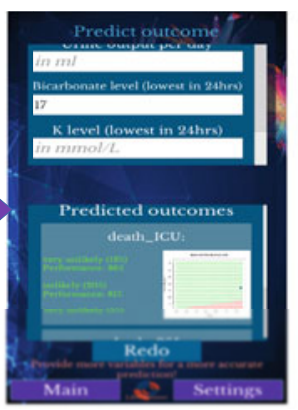

Example with 2 variables predicting "death after 1 year"

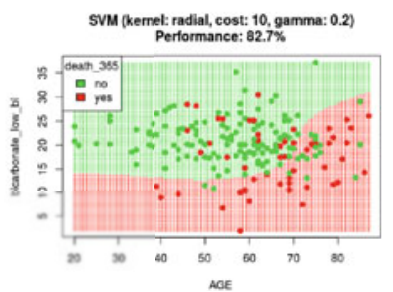

(e.g. 90 days mortality) of NSTI patients with high accuracy, supporting thereby patient stratification for differentiated treatment (LifeGlimmer $\mathrm{GmbH}$ )

validation cycles using enterprise-grade software-engineering methodologies and technologies. The CDSS prepares views for the clinicians and supports personnel with data that 
allow patient stratification and individualized therapy in NSTI/sepsis. In addition to individual patient data, the system provides information of patient classification into groups with similar disease status and comparable clinical and biological parameters, which are of great value for continued research. A simplified representation of the envisaged CDSS for NSTI is shown in Fig. 12.4.

An example of an initial prototype for a frontend app to be directly used by a clinical practitioner is depicted in Figs. 12.5 and 12.6 (www. lifeglimmer.com).

These modules are currently under development and testing and it is expected that they will be eventually implemented in the clinical practice, with potentially substantial benefits for the patient and disease management.

\subsection{Conclusion and Perspectives}

The clinical and biochemical research progress over the last decades have provided a burgeoning body of information on the possible mechanisms underlying NSTI, on the clinical manifestation of this fast-developing disease, and on individualized patient characteristics. The organization and translation of this information into actionable knowledge requires concerted, multidisciplinary efforts and accessible computational systems that assist decision making. Altogether, the efforts, platforms, and variety of modules herein described for systems and personalized medicine in acute infectious diseases form the basis for development of tools and concepts for refined diagnosis, patient stratification, and individualized treatment. Thereby, such approaches hold great promise for accurate and rapid diagnosing and improving outcome in NSTI, as well as potential to increase costefficacy, as it will promote optimized tailored therapy.

Acknowledgments The work was supported by the European Union Seventh Framework Programme: (FP7/2007-2013) under the grant agreement 305340 (INFECT project); the Swedish Governmental Agency for Innovation Systems (VINNOVA), Innovation Fund Denmark and the Research Council of Norway under the frame of NordForsk (Project no. 90456, PerAID), and the Swedish Research Council, Innovation Fund Denmark, the Research Council of Norway, the Netherlands Organisation for Health Research and Development (ZonMW), and DLR Federal Ministry of Education and Research, under the frame of ERA PerMed (Project 2018151, PerMIT).

\section{References}

Afzal M, Saccenti E, Madsen MB, Hansen MB, Hyldegaard O, Skrede S, Martins Dos Santos VAP, Norrby-Teglund A, Svensson M (2020) Integrated univariate, multivariate, and correlation-based network analyses reveal metabolite-specific effects on bacterial growth and biofilm formation in necrotizing soft tissue infections. J Proteome Res 19:688-698. https://doi. org/10.1021/acs.jproteome.9b00565

Ahn AC, Tewari M, Poon C-S, Phillips RS (2006) The limits of reductionism in medicine: could systems biology offer an alternative? PLoS Med 3:e208

Anaya DA, Mcmahon K, Nathens AB, Sullivan SR, Foy H, Bulger E (2005) Predictors of mortality and limb loss in necrotizing soft tissue infections. Arch Surg 140:151-157

Assfalg M, Bertini I, Colangiuli D, Luchinat C, Schafer H, Schutz B, Spraul M (2008) Evidence of different metabolic phenotypes in humans. Proc Natl Acad Sci USA 105:1420-1424

Auffray C, Charron D, Hood L (2010) Predictive, preventive, personalized and participatory medicine: back to the future. Genome Med 2(8):57. https://doi.org/10. 1186/gm178

Bahl M, Barzilay R, Yedidia AB, Locascio NJ, Yu L, Lehman CD (2018) High-risk breast lesions: a machine learning model to predict pathologic upgrade and reduce unnecessary surgical excision. Radiology 286:810-818

Bakker DJ (2012) Clostridial myonecrosis (gas gangrene). Undersea Hyperb Med 39:731

Bergsten H, Madsen MB, Bergey F, Hyldegaard O, Skrede S, Arnell P, Oppegaard O, Itzek A, Perner A, Svensson M (2020) Correlation between immunoglobulin dose administered and plasma neutralization of streptococcal superantigens in patients with necrotizing soft tissue infections. Clin Infect Dis 9:ciaa022

Bernini P, Bertini I, Luchinat C, Nepi S, Saccenti E, Scha Fer H, Schutz B, Spraul M, Tenori L (2009) Individual human phenotypes in metabolic space and time. J Proteome Res 8:4264-4271

Berthold MR, Borgelt C, Höppner F, Klawonn F (2010) Guide to intelligent data analysis: how to intelligently make sense of real data. Springer, New York

Białecki A, Muir R, Ingersoll G (2012) Apache lucene 4. SIGIR 2012 workshop on open source information retrieval, p 17 
Bosshardt TL, Henderson VJ, Organ CH (1996) Necrotizing soft-tissue infections. Arch Surg 131:846-854

Bresnick J (2016) The difference between big data and smart data in healthcare. Health Anal. https:// healthitanalytics.com/features/the-difference-betweenbig-data-and-smart-data-in-healthcare. Accessed May 2020

Bresnick J (2018) What are precision medicine and personalized medicine? Health analytics [online]. https://healthitanalytics.com/features/whatareprecision-medicine-and-personalized-medicine. Accessed May 2020

Brighi R (2018) The quality and veracity of digital data on health: from electronic health records to big data. Revista de Bioética y Derecho 42:163-179

Chella Krishnan K, Mukundan S, Alagarsamy J, Hur J, Nookala S, Siemens N, Svensson M, Hyldegaard O, Norrby-Teglund A, Kotb M (2016) Genetic architecture of group A streptococcal necrotizing soft tissue infections in the mouse. PLoS Pathog 12:e1005732

Clark MM, Hildreth A, Batalov S, Ding Y, Chowdhury S, Watkins K, Ellsworth K, Camp B, Kint CI, Yacoubian C, Farnaes L, Bainbridge MN, Beebe C, Braun JJA, Bray M, Carroll J, Cakici JA, Caylor SA, Clarke C, Creed MP, Friedman J, Frith A, Gain R, Gaughran M, George S, Gilmer S, Gleeson J, Gore J, Grunenwald H, Hovey RL, Janes ML, Lin K, McDonagh PD, McBride K, Mulrooney P, Nahas S, Oh D, Oriol A, Puckett L, Rady Z, Reese MG, Ryu J, Salz L, Sanford E, Stewart L, Sweeney N, Tokita M, Van der Kraan L, White S, Wigby K, Williams B, Wong T, Wright MS, Yamada C, Schols P, Reynders J, Hall K, Dimmock D, Veeraraghavan N, Defay T, Kingsmore SF (2019) Diagnosis of genetic diseases in seriously ill children by rapid wholegenome sequencing and automated phenotyping and interpretation. Sci Transl Med 11:6177

Cote RA (1986) Architecture of SNOMED: its contribution to medical language processing. Proceedings of the annual symposium on computer application in medical care, American Medical Informatics Association, p 74

Davenport EE, Burnham KL, Radhakrishnan J, Humburg P, Hutton P, Mills TC, Rautanen A, Gordon AC, Garrard C, Hill AV (2016) Genomic landscape of the individual host response and outcomes in sepsis: a prospective cohort study. Lancet Respir Med 4:259-271

Dias D, Paulo Silva Cunha J (2018) Wearable health devices-vital sign monitoring, systems and technologies. Sensors 18:2414

Erikainen S, Chan S (2019) Contested futures: envisioning "Personalized," "Stratified," and "Precision" medicine. New Genet Soc 38:308-330

Esteva A, Kuprel B, Novoa RA, Ko J, Swetter SM, Blau HM, Thrun S (2017) Dermatologist-level classification of skin cancer with deep neural networks. Nature 542:115-118
European Commission (2016) Guidelines on FAIR data management in horizon 2020. https://www.google.com/ url?sa $=\mathrm{t} \& \mathrm{rct}=\mathrm{j} \& \mathrm{q}=\& \mathrm{esrc}=\mathrm{s} \&$ source $=$ web $\& \mathrm{~cd}=\& \mathrm{ved}=$ 2ahUKEwjOrb3ZgNTrAhWQmIsKHQ2xBhsQFjAAe gQIAhAB\&url=https $\% 3 \mathrm{~A} \% 2 \mathrm{~F} \% 2 \mathrm{Fec}$.europa.eu $\%$ 2Fresearch $\% 2$ Fparticipants $\% 2$ Fdata $\% 2$ Fref $\%$ 2Fh2020\%2Fgrants_manual\%2Fhi\%2Foa_pilot $\%$ 2Fh2020-hi-oa-datamgt_en.pdf\&usg= AOvVaw0RrG7kc_LE3Hp74lceZDA5

Fernando SM, Tran A, Cheng W, Rochwerg B, Kyeremanteng K, Seely AJ, Inaba K, Perry JJ (2019) Necrotizing soft tissue infection: diagnostic accuracy of physical examination, imaging, and LRINEC score: a systematic review and meta-analysis. Ann Surg 269:58-65

Frost \& Sullivan (2015) Drowning in big data? Reducing information technology complexities and costs for healthcare organizations, Frost \& Sullivan [Online]. https://www.emc.com/collateral/analyst-reports/frostsullivan-reducing-information-technologycomplexities-ar.pdf. Accessed 7 May 2017

Ganzinger M, Knaup P (2017) Information management for enabling systems medicine. Curr Direct Biomed Eng 3:501-504

Garg AX, Adhikari NK, McDonald H, Rosas-Arellano MP, Devereaux PJ, Beyene J, Sam J, Haynes RB (2005) Effects of computerized clinical decision support systems on practitioner performance and patient outcomes: a systematic review. JAMA 293:1223-1238

Goh T, Goh L, Ang C, Wong C (2014) Early diagnosis of necrotizing fasciitis. Br J Surg 101:e119-e125

Goldstein EJ, Anaya DA, Dellinger EP (2007) Necrotizing soft-tissue infection: diagnosis and management. Clin Infect Dis 44:705-710

Gruden K, Hren M, Herman A, Blejec A, Albrecht T, Selbig J, Bauer C, Schuchardt J, Or-Guil M, Zupančič K (2012) A "crossomics" study analysing variability of different components in peripheral blood of healthy caucasoid individuals. PLoS One 7:e28761

Hakkarainen TW, Kopari NM, Pham TN, Evans HL (2014) Necrotizing soft tissue infections: review and current concepts in treatment, systems of care, and outcomes. Curr Probl Surg 51:344

Hansen MB, Rasmussen LS, Svensson M, Chakrakodi B, Bruun T, Madsen MB, Perner A, Garred P, Hyldegaard O, Norrby-Teglund A, INFECT Study Group, Nekludov M, Arnell P, Rosén A, Oscarsson N, Karlsson Y, Oppegaard O, Skrede S, Itzek A, Wahl AM, Hedetoft M, Bærnthsen NF, Müller R, Nedrebø T (2017) Association between cytokine response, the LRINEC score and outcome in patients with necrotising soft tissue infection: a multicentre, prospective study. Sci Rep 7:42179

Hietbrink F, Bode LG, Riddez L, Leenen LPH, Van Dijk MR (2016) Triple diagnostics for early detection of ambivalent necrotizing fasciitis. World J Emerg Surg 11:51

Hotchkiss RS, Karl IE (2003) The pathophysiology and treatment of sepsis. N Engl J Med 348:138-150 
Hsiao C-T, Chang C-P, Huang T-Y, Chen Y-C, Fann W-C (2020) Prospective validation of the laboratory risk indicator for necrotizing fasciitis (LRINEC) score for necrotizing fasciitis of the extremities. PLoS One 15: e0227748

Hua C, Sbidian E, Hemery F, Decousser JW, Bosc R, Amathieu R, Rahmouni A, Wolkenstein P, ValeyrieAllanore L, Brun-Buisson C (2015) Prognostic factors in necrotizing soft-tissue infections (NSTI): a cohort study. J Am Acad Dermatol 73:1006-1012

Huang K-F, Hung M-H, Lin Y-S, Lu C-L, Liu C, Chen C-C, Lee Y-H (2011) Independent predictors of mortality for necrotizing fasciitis: a retrospective analysis in a single institution. J Trauma Acute Care Surg 71:467-473

Hughes DA, Kircher M, He Z, Guo S, Fairbrother GL, Moreno CS, Khaitovich P, Stoneking M (2015) Evaluating intra-and inter-individual variation in the human placental transcriptome. Genome Biol 16:54

Hulsen T, Jamuar SS, Moody A, Karnes JH, Orsolya V, Hedensted S, Spreafico R, Hafler DA, McKinney EF (2019) From big data to precision medicine. Front Med $6: 34$

Jolliffe I (1982) A note on the use of principal components in regression. J Roy Stat Soc C-App 31:300-303

Kim K-T, Kim YJ, Won Lee J, Kim YJ, Park S-W, Lim MK, Suh CH (2011) Can necrotizing infectious fasciitis be differentiated from nonnecrotizing infectious fasciitis with MR imaging? Radiology 259:816-824

Kittang BR, Langeland N, Skrede S, Mylvaganam H (2010) Two unusual cases of severe soft tissue infection caused by Streptococcus dysgalactiae subsp. equisimilis. J Clin Microbiol 48:1484-1487

Komorowski M (2020) Clinical management of sepsis can be improved by artificial intelligence: yes. Intensive Care Med 46:375-377

Lassila O, Swick RR (1998) Resource description framework (RDF) model and syntax specification. World Wide Web Consortium, Cambridge

Lazăr A, Georgescu AM, Vitin A, Azamfirei L (2019) Precision medicine and its role in the treatment of sepsis: a personalised view. J Crit Care Med 5:90-96

Leichtle SW, Tung L, Khan M, Inaba K, Demetriades D (2016) The role of radiologic evaluation in necrotizing soft tissue infections. J Trauma Acute Care Surg 81:921-924

Lussier YA, Rothwell D, Cote R (1998) The SNOMED model: a knowledge source for the controlled terminology of the computerized patient record. Methods Inf Med 37:161-164

Madsen MB, Skrede S, Bruun T, Arnell P, Rosén A, Nekludov M, Karlsson Y, Bergey F, Saccenti E, Martins dos Santos VAP, Perner A, Norrby-Teglund A, Hyldegaard O (2018) Necrotizing soft tissue infections - a multicentre, prospective observational study (INFECT): protocol and statistical analysis plan. Acta Anaesthesiol Scand 62:272-279

Madsen MB, Skrede S, Perner A, Arnell P, Nekludov M, Bruun T, Karlsson Y, Hansen MB, Polzik P, Hedetoft
M (2019) Patient's characteristics and outcomes in necrotising soft-tissue infections: results from a Scandinavian, multicentre, prospective cohort study. Intensive Care Med 45:1241-1251

Magrabi F, Ammenwerth E, Hyppönen H, de Keizer N, Nykänen P, Rigby M, Scott P, Talmon J, Georgiou A (2016) Improving evaluation to address the unintended consequences of health information technology. Yearb Med Inform 25:61-69

Mamlin BW, Tierney WM (2016) The promise of information and communication technology in healthcare: extracting value from the chaos. Am J Med Sci 351:59-68

Meier-Schellersheim M, Fraser ID, Klauschen F (2009) Multiscale modeling for biologists. Wiley Interdiscip Rev Syst Biol Med 1:4-14

Mills MK, Faraklas I, Davis C, Stoddard GJ, Saffle J (2010) Outcomes from treatment of necrotizing softtissue infections: results from the National Surgical Quality Improvement Program database. Am J Surg 200:790-797

Morgan M (2010) Diagnosis and management of necrotising fasciitis: a multiparametric approach. J Hosp Infect 75:249-257

Nicholson JK, Holmes E, Kinross JM, Darzi AW, Takats Z, Lindon JC (2012) Metabolic phenotyping in clinical and surgical environments. Nature 491:384-392

Nimmesgern E, Benediktsson I, Norstedt I (2017) Personalized medicine in Europe. Clin Transl Sci 10:61-63

Noble D (2008) Claude Bernard, the first systems biologist, and the future of physiology. Exp Physiol 93:16-26

Patcas R, Bernini DA, Volokitin A, Agustsson E, Rothe R, Timofte R (2019) Applying artificial intelligence to assess the impact of orthognathic treatment on facial attractiveness and estimated age. Int J Oral Maxillofac Surg 48:77-83

Peetermans M, de Prost N, Eckmann C, Norrby-TeglundA, Skrede S, de Waele JJ (2020) Necrotizing skin and soft-tissue infections in the intensive care unit. Clin Microbiol Infect 26:8-17

Phillips M, Marsden H, Jaffe W, Matin RN, Wali GN, Greenhalgh J, Mcgrath E, James R, Ladoyanni E, Bewley A, Argenziano G, Palamaras I (2019) Assessment of accuracy of an artificial intelligence algorithm to detect melanoma in images of skin lesions. JAMA Netw Open 2:e1913436-e1913436

Phillips M, Greenhalgh J, Marsden H, Palamaras I (2020) Detection of malignant melanoma using artificial intelligence: an observational study of diagnostic accuracy. Dermatology Practical \& Conceptual 10:e2020011

Pusic M, Ansermino M (2004) Clinical decision support systems. B C Med J 46:236-239

Rajpurkar P, Irvin J, Zhu K, Yang B, Mehta H, Duan T, Ding D, Bagul A, Langlotz C, Shpanskaya K (2017) Chexnet: radiologist-level pneumonia detection on chest X-rays with deep learning. Preprint arXiv: 1711.05225 
Rakus-Andersson E, Frey J (2016) Fuzzy one-decision making model with fuzzified outcomes in the treatment of necrotizing fasciitis, eTELEMED, Venice, Italy, 2016. International Academy, Research and Industry Association (IARIA), pp 145-152

Regulation GDP (2016) Regulation (EU) 2016/679 of the European Parliament and of the council of 27 April 2016 on the protection of natural persons with regard to the processing of personal data and on the free movement of such data, and repealing directive 95/46. Official J European Union 59:294

Rello J, Van Engelen T, Alp E, Calandra T, Cattoir V, Kern W, Netea M, Nseir S, Opal S, Van de Veerdonk F (2018) Towards precision medicine in sepsis: a position paper from the European Society of Clinical Microbiology and Infectious Diseases. Clin Microbiol Infect 24:1264-1272

Rosato A, Tenori L, Cascante M, de Atauri Carulla PR, Martins dos Santos VAP, Saccenti E (2018) From correlation to causation: analysis of metabolomics data using systems biology approaches. Metabolomics $14: 37$

Sarani B, Strong M, Pascual J, Schwab CW (2009) Necrotizing fasciitis: current concepts and review of the literature. J Am Coll Surg 208:279-288

Schroeck M, Shockley R, Smart J, Romero-Morales D, Tufano P (2012) Analytics: the real-world use of big data: how innovative enterprises extract value from uncertain data, Executive Report. IBM Institute for Business Value and Said Business School at the University of Oxford

Smith KP, Wang H, Durant TJ, Mathison BA, Sharp SE, Kirby JE, Long SW, Rhoads DD (2020) Applications of artificial intelligence in clinical microbiology diagnostic testing. Clin Microbiol News1 42:61-70

Stacy A, McNally L, Darch SE, Brown SP, Whiteley M (2016) The biogeography of polymicrobial infection. Nat Rev Microbiol 14:93

Stevens DL, Bryant AE (2017) Necrotizing soft-tissue infections. N Engl J Med 377:2253-2265
Sutton RT, Pincock D, Baumgart DC, Sadowski DC, Fedorak RN, Kroeker KI (2020) An overview of clinical decision support systems: benefits, risks, and strategies for success. NPJ Digital Med 3:1-10

Thänert R, Itzek A, Hoßmann J, Hamisch D, Madsen MB, Hyldegaard O, Skrede S, Bruun T, Norrby-Teglund A, Medina E (2019) Molecular profiling of tissue biopsies reveals unique signatures associated with streptococcal necrotizing soft tissue infections. Nat Commun 10:1-15

Tillmann T, Gibson RA, Scott G, Harrison O, Dominiczak A, Hanlon P (2015) Systems medicine 2.0: potential benefits of combining electronic health care records with systems science models. J Med Internet Res 17:e64

Tøndel K, Indahl UG, Gjuvsland AB, Omholt SW, Martens H (2012) Multi-way metamodelling facilitates insight into the complex input-output maps of nonlinear dynamic models. BMC Syst Biol 6:88

Union C. O. T. E (2015) Council conclusions on personalised medicine for patients. Official $\mathrm{J}$ European Union 421:2

van Dam JC, Koehorst JJ, Schaap PJ, Martins dos Santos VA, Suarez-DIEZ M (2015) RDF2Graph a tool to recover, understand and validate the ontology of an RDF resource. J Biomed Semant 6:1-12

Vitabile S, Marks M, Stojanovic D, Pllana S, Molina JM, Krzyszton M, Sikora A, Jarynowski A, Hosseinpour F, Jakobik A (2019) Medical data processing and analysis for remote health and activities monitoring. In: Highperformance modelling and simulation for big data applications. Springer, Cham

Wasylewicz A, Scheepers-Hoeks A (2019) Clinical decision support systems. In: Fundamentals of clinical data science. Springer, Cham

Wold S, Eriksson L (2001) PLS-regression: a basic tool of chemometrics. Chemom Intel Lab Syst 58:109-130

Wong C-H, Khin L-W, Heng K-S, Tan K-C, Low C-O (2004) The LRINEC (laboratory risk indicator for necrotizing fasciitis) score: a tool for distinguishing necrotizing fasciitis from other soft tissue infections. Crit Care Med 32:1535-1541 